\title{
Itô formula for processes taking values in intersection of finitely many Banach spaces
}

\author{
István Gyöngy ${ }^{1}$ • David Šiška ${ }^{1}$
}

Received: 5 September 2016 / Published online: 17 March 2017

(C) The Author(s) 2017. This article is an open access publication

Abstract Motivated by applications to SPDEs we extend the Itô formula for the square of the norm of a semimartingale $y(t)$ from Gyöngy and Krylov (Stochastics $6(3): 153-173,1982)$ to the case

$$
\sum_{i=1}^{m} \int_{(0, t]} v_{i}^{*}(s) d A(s)+h(t)=: y(t) \in V \quad d A \times \mathbb{P} \text {-a.e., }
$$

where $A$ is an increasing right-continuous adapted process, $v_{i}^{*}$ is a progressively measurable process with values in $V_{i}^{*}$, the dual of a Banach space $V_{i}, h$ is a cadlag martingale with values in a Hilbert space $H$, identified with its dual $H^{*}$, and $V:=V_{1} \cap V_{2} \cap \cdots \cap V_{m}$ is continuously and densely embedded in $H$. The formula is proved under the condition that $\|y\|_{V_{i}}^{p_{i}}$ and $\left\|v_{i}^{*}\right\|_{V_{i}^{*}}^{q_{i}}$ are almost surely locally integrable with respect to $d A$ for some conjugate exponents $p_{i}, q_{i}$. This condition is essentially weaker than the one which would arise in application of the results in Gyöngy and Krylov (Stochastics 6(3):153-173, 1982) to the semimartingale above.

Keywords Stochastic evolution equations - Stochastic partial differential equations · Itô's formula $\cdot$ Energy equality

David Šiška

d.siska@ed.ac.uk

István Gyöngy

i.gyongy@ed.ac.uk

1 School of Mathematics, University of Edinburgh, Edinburgh, UK 


\section{Introduction}

Itô formula for the square of the norm is an essential tool in the study of stochastic evolution equations of the type

$$
d v(t)=\mathbb{A}(t, v(t)) d t+\sum_{k} \mathbb{B}_{k}(t, v(t)) d W^{k}(t)
$$

where $\left(W^{k}\right)_{k=1}^{\infty}$ is a sequence of independent Wiener processes, and $\mathbb{A}(t, \cdot)$ and $\mathbb{B}_{k}(t, \cdot)$ are (possibly random nonlinear) operators on a separable real Banach space $V$, with values in a Banach space $V^{\prime}$ and a Hilbert space $H$ respectively, such that $V \hookrightarrow H \hookrightarrow$ $V^{\prime}$ with continuous and dense embeddings. We assume there is a constant $K$ such that $(v, h) \leq K\|v\|_{V}\|h\|_{V^{\prime}}$ for all $v \in V$ and $h \in H$. This means that for the linear mapping $\Psi: H \rightarrow H^{*}$, which identifies $H$ with its dual $H^{*}$ via the inner product in $H$, we have $\|\Psi(h)\|_{V^{*}} \leq K\|h\|_{V^{\prime}}$. Therefore, since $H$ is dense in $V^{\prime}, \Psi$ can be extended to a continuous mapping from $V^{\prime}$ into $V^{*}$, the dual of $V$. It is assumed that this extension is one-to-one from $V^{\prime}$ into $V^{*}$. Thus an initial value problem for Eq. (1.1) can be viewed as

$$
v(t)=\int_{0}^{t} v^{*}(s) d s+h(t)=: y(t)
$$

with the $V^{*}$-valued process $v^{*}(t):=\mathbb{A}(t, v(t))$ and $H \equiv H^{*}$-valued process

$$
h(t):=h_{0}+\sum_{k} \int_{0}^{t} \mathbb{B}^{k}(s, v(s)) d W^{k}(s),
$$

where $h_{0}$ is a given initial value and the equality (1.2) in $V^{*}$ is required $d t \times \mathbb{P}$ almost everywhere. In the special case $B_{k}=0$ for every $k$, and nonrandom $h_{0}$ and $A$, i.e., in the case

$$
v(t)=h_{0}+\int_{0}^{t} v^{*}(s) d s, \quad d t \text {-a.e. }
$$

it is well-known that when $v \in L_{p}([0, T], V), v^{*} \in L_{q}\left([0, T], V^{*}\right)$ for $T>0$ and conjugate exponents $p$ and $q$, then there is $u \in C([0, T], H)$ such that $u=v$ for $d t$-almost all $t \in[0, T]$ and the "energy equality"

$$
|u(t)|_{H}^{2}=\left|h_{0}\right|_{H}^{2}+2 \int_{0}^{t}\left\langle v^{*}(s), v(s)\right\rangle d s
$$

holds for all $t \in[0, T]$, where $\langle\cdot, \cdot\rangle$ denotes the duality pairing of $V^{*}$ and $V$. This formula is used in proofs of existence and uniqueness theorems for PDEs, see e.g., [3] and [13]. A generalisation of it, a "stochastic energy equality", i.e., an Itô formula for the square of the $H$-norm of $y$, was first presented in Pardoux [14], and was used to obtain existence and uniqueness theorems for SPDEs. The proof of it in [14] was not 
separated from the theory of SPDEs developed there. A proof, not bound to the theory of SPDEs, was given in Krylov and Rozovskii [12], and then this stochastic energy equality was generalised in Gyöngy and Krylov [6] to $V^{*}$-valued semimartingales $y$ of the form

$$
y(t)=\int_{(0, t]} v^{*}(s) d A(s)+h(t),
$$

where $A$ is an adapted nondecreasing cadlag process and $h$ is an $H$-valued cadlag martingale. This generalisation is used in Gyöngy [7] to extend the theory of SPDEs developed in [14] and [12] to SPDEs driven by random orthogonal measures and Lévy martingales, written in the form

$$
d v(t)=\mathbb{A}(t, v(t)) d A(t)+\mathbb{B}(t, v(t)) d M(t)
$$

with cadlag (quasi left-continuous) martingales $M$ with values in a Hilbert space.

In the present paper we are interested in stochastic energy equalities which can be applied to SPDEs (1.4) when $\mathbb{A}$ is of the form $\mathbb{A}=\mathbb{A}_{1}+\mathbb{A}_{2}+\cdots+\mathbb{A}_{m}$ and the operators $\mathbb{A}_{i}$ have different analytic and growth properties. This means,

$$
\mathbb{A}_{i}(t, \cdot): V_{i} \rightarrow V_{i}^{\prime} \quad i=1,2, \ldots, m
$$

for some Banach spaces $V_{i}$ and $V_{i}^{\prime}$, such that with a constant $R$ and a process $g$, locally integrable with respect to $d A$, one has for all $t$

$$
\left\|\mathbb{A}_{i}(t, w)\right\|_{V_{i}^{\prime}} \leq\left|g_{t}\right|^{1 / q_{i}}+R\|w\|_{V_{i}}^{p_{i}-1}
$$

for all $w \in V, q_{i}=p_{i} /\left(p_{i}-1\right)$ with (possibly) different exponents $p_{i} \geq 1$, which for $p_{i}=1$ means that $\left\|\mathbb{A}_{i}(t, w)\right\|_{V_{i}^{\prime}}$ is bounded by a constant.

In the special case when $A(t)=t$ and $M$ is a Wiener process the above situation was considered in [14], and a related stochastic energy equality was also presented there. Our main result, Theorem 2.1 generalises the results on stochastic energy equalities from [14] and [6]. We prove it by adapting the method of the proof of the main theorem in $[6]$.

In the present paper we consider a semimartingale $y$ of the form (1.3) such that $d A \times \mathbb{P}$-almost everywhere $y$ takes values in $V=V_{1} \cap \ldots \cap V_{m}$, where $V_{i}$ are Banach spaces (over $\mathbb{R}$ ) such that $V$ with the norm $\|\cdot\|:=\sum_{i=1}^{m}\|\cdot\|_{V_{i}}$ is continuously and densely embedded in $H$. The process $v^{*}$ in (1.3) is of the form $v^{*}=\sum_{i=1}^{m} v_{i}^{*}$, where $v_{i}^{*}$ are $V_{i}^{*}$-valued progressively measurable processes. We prove that $y$ is almost surely cadlag as a process with values in $H$ and for $|y|_{H}^{2}$ an Itô formula holds under the assumption that $\|y\|_{V_{i}}^{p_{i}}$ and $\left\|v_{i}^{*}\right\|_{V_{i}^{*}}^{q_{i}}$ are almost surely locally integrable with respect to $d A$ for some conjugate exponents $p_{i}, q_{i}$. See Sect. 2 for precise formulation of the main theorem. To apply the result of [6] to $y$ given by (1.3), one needs the local integrability (with respect to $d A$ ) of

$$
\|y\|_{V}\left\|v^{*}\right\|_{V^{*}}=\left(\|y\|_{V_{1}}+\cdots+\|y\|_{V_{m}}\right)\left\|v_{1}^{*}+\cdots+v_{m}^{*}\right\|_{V^{*}},
$$


which, in general, is not satisfied under our assumptions. See Remark 2.1 and Example 2.1 .

We note that in the context of stochastic evolution equations it is possible to prove Itô formulae for more general functions (satisfying appropriate differentiability assumptions), see again Pardoux [14], Krylov [9-11], Da Prato et al. [1], as well as Dareiotis and Gyöngy [2]. The Itô formula for the square of the norm is used in particular to establish a priori estimates as well as uniqueness and existence of solutions of stochastic evolution equations. The more general Itô formula can then be used to study finer properties of solutions of stochastic evolution equations, for example the maximum principle.

For general theory of SPDEs in the variational setting we refer the reader to Krylov and Rozovskii [12], Prévôt and Röckner [15] and Rozovskii [16].

\section{Main results}

For $i=1, \ldots, m$ let $\left(V_{i},\|\cdot\|_{V_{i}}\right)$ be real Banach spaces with duals $\left(V_{i}^{*},\|\cdot\|_{V_{i}^{*}}\right)$. Let $V$ denote the vector space $V_{1} \cap \cdots \cap V_{m}$ with the norm $\|\cdot\|:=\|\cdot\| V_{1}+\cdots+\|\cdot\|_{V_{m}}$. Then clearly, $V$ is a Banach space. Assume that it is separable and is continuously and densely embedded in a Hilbert space $(H,|\cdot|)$, which is identified with its dual $H^{*}$ by the help of the inner product $(\cdot, \cdot)$ in $H$. Thus we have

$$
V \hookrightarrow H \equiv H^{*} \hookrightarrow V^{*}
$$

where $H^{*} \hookrightarrow V^{*}$ is the adjoint of the embedding $V \hookrightarrow H$. We use the notation $\langle\cdot, \cdot\rangle$ for the duality pairing between $V$ and $V^{*}$. Note that if $v^{*} \in V_{i}^{*}$ for some $i$, then its restriction to $V$ belongs to $V^{*}$ and $\left|\left\langle v^{*}, v\right\rangle\right| \leq\left\|v^{*}\right\|_{V_{i}^{*}}\|v\|_{V_{i}}$ for all $v \in V$. Note also that $\left\langle v^{*}, v\right\rangle=(h, v)$ for for all $v \in V$ when $v^{*}=h \in H$.

A complete probability space $(\Omega, \mathcal{F}, \mathbb{P})$ together with an increasing family of $\sigma$ algebras $\left(\mathcal{F}_{t}\right)_{t \geq 0}, \mathcal{F}_{t} \subset \mathcal{F}$ will be used throughout the paper. Moreover it is assumed that the usual conditions are satisfied: $\bigcap_{s>t} \mathcal{F}_{s}=\mathcal{F}_{t}$ and $\mathcal{F}_{0}$ contains all subsets of $\mathbb{P}$-null sets of $\mathcal{F}$. We use the notation $\mathcal{B}\left(\mathbb{R}_{+}\right)$for the $\sigma$-algebra of Borel subsets of $\mathbb{R}_{+}=[0, \infty)$, and for a real-valued increasing $\mathcal{B}\left(\mathbb{R}_{+}\right) \otimes \mathcal{F}$-measurable process $(A(t))_{t \geq 0}$ the notation $d A \times \mathbb{P}$ stands for the measure defined on $\mathcal{B}\left(\mathbb{R}_{+}\right) \otimes \mathcal{F}$ by

$$
(d A \times \mathbb{P})(F)=\mathbb{E} \int_{0}^{\infty} \mathbf{1}_{F} d A(t), \quad F \in \mathcal{B}\left(\mathbb{R}_{+}\right) \otimes \mathcal{F}
$$

Let $h=(h(t))_{t \geq 0}$ be an $H$-valued locally square integrable martingale that is cadlag (continuous from the right with left-hand limits) in the strong topology on $H$. Its quadratic variation process is denoted by $[h]$, and $\langle h\rangle$ denotes the unique predictable process starting from zero such that $|h|^{2}-\langle h\rangle$ is a local martingale. Furthermore let $A$ be a real-valued nondecreasing adapted cadlag process starting from zero. Finally let $v=$ $(v(t))_{t \geq 0}$ be a $V$-valued progressively measurable process and for $i=1, \ldots, m$ let $v_{i}^{*}=\left(v_{i}^{*}\right)_{t \geq 0}$ be $V_{i}^{*}$-valued processes such that $\left\langle\varphi, v_{i}^{*}\right\rangle$ are progressively measurable 
for any $\varphi \in V$. Notice that $v$ is also progressively measurable as a process with values in $\bar{V}_{i}$, the closure in $V_{i}$-norm of the linear hull of $\{v(t): t \geq 0, \omega \in \Omega\}$.

Let there be $p_{i} \in[1, \infty)$ and $q_{i}=p_{i} /\left(p_{i}-1\right) \in(1, \infty]$, where, as usual, $1 / 0:=\infty$. Assume that for each $i=1,2, \ldots, m$ and $T>0$

$$
\int_{0}^{T}\|v(t)\|_{V_{i}}^{p_{i}} d A(t)<\infty, \quad\left(\int_{0}^{T} \eta_{i}^{q_{i}}(t) d A(t)\right)^{1 / q_{i}}<\infty,
$$

for some progressively measurable process $\eta_{i}$ such that $\left\|v_{i}^{*}\right\|_{V_{i}^{*}} \leq \eta_{i}$ for $d A \times \mathbb{P}$ almost everywhere, where for $q_{i}=\infty$ the second expression means

$$
d A \text {-ess } \sup _{t \leq T} \eta_{i}(t)
$$

the essential supremum (with respect to $d A$ ) of $\eta_{i}$ over [0,T].

The following theorem is the main result of this paper.

Theorem 2.1 Let $\tau$ be a stopping time. Suppose that for all $\varphi \in V$ and for $d A \times \mathbb{P}$ almost all $(\omega, t)$ such that $t \in(0, \tau(\omega))$ we have

$$
(v(t), \varphi)=\sum_{i=1}^{m} \int_{(0, t]}\left\langle v_{i}^{*}(s), \varphi\right\rangle d A(s)+(h(t), \varphi) .
$$

Then there is $\tilde{\Omega} \subset \Omega$ with $\mathbb{P}(\tilde{\Omega})=1$ and an $H$-valued cadlag process $\tilde{v}$ such that the following statements hold.

(i) For $d A \times \mathbb{P}$ almost all $(t, \omega)$ satisfying $t \in(0, \tau(\omega))$ we have $\tilde{v}=v$.

(ii) For all $\omega \in \tilde{\Omega}$ and $t \in[0, \tau(\omega))$ we have

$$
(\tilde{v}(t), \varphi)=\sum_{i=1}^{m} \int_{(0, t]}\left\langle v_{i}^{*}(s), \varphi\right\rangle d A(s)+h(t) \varphi \text { for all } \varphi \in V .
$$

(iii) For all $\omega \in \tilde{\Omega}$ and $t \in[0, \tau(\omega))$

$$
\begin{aligned}
|\tilde{v}(t)|^{2}= & |h(0)|^{2}+2 \sum_{i=1}^{m} \int_{(0, t]}\left\langle v_{i}^{*}(s), v(s)\right\rangle d A(s)+2 \int_{(0, t]}(\tilde{v}(s-) d h(s)) \\
& -\int_{(0, t]}\left|v^{*}(s)\right|^{2} \Delta A(s) d A(s)+[h]_{t},
\end{aligned}
$$

where $v^{*}(t):=\sum_{i=1}^{m} v_{i}^{*}(t) \in H$ for $\Delta A(t)>0$.

Consider now a situation where the assumptions on $h$ and $A$ are as above but $m=1$ and regarding $v$ and $v^{*}:=v_{1}^{*}$ we know that $\|v(t)\|,\left\|v^{*}(t)\right\|_{V^{*}}$ and $\|v(t)\|\left\|v^{*}(t)\right\|_{V^{*}}$ are almost surely locally integrable with respect to $d A(t)$. Let

$$
\bar{v}^{*}(t):=\frac{v^{*}(t)}{1+\left\|v^{*}(t)\right\|_{V^{*}}} \text { and } \bar{A}(t):=\int_{(0, t]}\left(1+\left\|v^{*}(t)\right\|_{V^{*}}\right) d A(t) .
$$


Then $\left\|\bar{v}^{*}\right\|_{V^{*}} \leq 1$ and so $v, \bar{v}^{*}$ and $\bar{A}$ satisfy the conditions on $v, v^{*}$ and $A$, respectively, with $p_{1}=1$ and $q_{1}=\infty$. If (2.2) holds for all $\varphi \in V$ and for $d A \times \mathbb{P}$ almost all $(\omega, t)$ such that $t \in(0, \tau(\omega))$ then

$$
(v(t), \varphi)=\sum_{i=1}^{m} \int_{(0, t]}\left\langle\bar{v}^{*}(s), \varphi\right\rangle d \bar{A}(s)+(h(t), \varphi)
$$

Applying Theorem 2.1 then means that we have all of its conclusions with $\bar{v}^{*}$ and $\bar{A}$ in place of $v^{*}$ and $A$ respectively. In particular, we get

$$
\begin{aligned}
|\tilde{v}(t)|^{2}= & |h(0)|^{2}+2 \int_{(0, t]}\left\langle\bar{v}^{*}(s), v(s)\right\rangle d \bar{A}(s)+2 \int_{(0, t]}(\tilde{v}(s-), d h(s)) \\
& -\int_{(0, t]}\left|\bar{v}^{*}(s)\right|^{2} \Delta \bar{A}(s) d \bar{A}(s)+[h]_{t} \\
= & |h(0)|^{2}+2 \int_{(0, t]}\left\langle v^{*}(s), v(s)\right\rangle d A(s)+2 \int_{(0, t]}(\tilde{v}(s-), d h(s)) \\
& -\int_{(0, t]}\left|v^{*}(s)\right|^{2} \Delta A(s) d A(s)+[h]_{t} .
\end{aligned}
$$

Hence we see that Theorem 2.1 is a generalisation of the main theorem in Gyöngy and Krylov [6].

Remark 2.1 One might think that Theorem 2.1 follows from the main theorem in [6] by considering the process $v^{*}=\sum_{i} v_{i}^{*}$ as a process with values in $V^{*}$. However, taking into account that for any $w^{*} \in V^{*}$

$$
\left\|w^{*}\right\|_{V^{*}}=\inf \left\{\max _{i=1, \ldots, m}\left\|w_{i}^{*}\right\|_{V_{i}^{*}}: w^{*}=\sum_{i=1}^{m} w_{i}^{*}, w_{i}^{*} \in V_{i}^{*}\right\}
$$

(see for example Gajewski, Gröger and Zacharias [4, Chapter 1, Theorem 5.13]), one can show that the local integrability condition in [6] for

$$
\|v\|_{V}\left\|v^{*}\right\|_{V^{*}}=\left(\|v\|_{1}+\cdots+\|v\|_{m}\right)\left\|v^{*}\right\|_{V^{*}}
$$

is not implied by our assumption (2.1). Thus the main theorem in [6] is not applicable in our situation.

We consider the following motivating example.

Example 2.1 Consider the stochastic partial differential equation

$$
\begin{aligned}
d u= & {\left[\nabla\left(|\nabla u|^{p_{1}-2} \nabla u\right)+|u|^{p_{2}-2} u\right] d t } \\
& +f(u, \nabla u) d W+\int_{Z} g(u) q(d t, d z) \text { in } \mathscr{D} \times(0, T) .
\end{aligned}
$$


Here $W$ is a Wiener process (finite or infinite dimensional depending on the choice of $f),(Z, \Sigma)$ is a measurable space and $q(d s, d z)$ a stochastic martingale measure on $[0, \infty) \times Z$. See, for example, Gyöngy and Krylov [5] for detailed definition. We take $\mathscr{D}$ to be a bounded Lipschitz domain in $\mathbb{R}^{d}$.

It is natural to assume that a solution $u$ should be such that $\|u\|_{W_{p_{1}}^{1}(\mathscr{D})}^{p_{1}}$ and $\|u\|_{L_{p_{2}}(\mathscr{D})}^{p_{2}}$ are almost surely locally integrable. To apply the result in Gyöngy and Krylov [6] one could try to take $V:=W_{p_{1}}^{1}(\mathscr{D}) \cap L_{p_{2}}(\mathscr{D})$ with the norm $\|\cdot\|_{V}=\|\cdot\|_{W_{p_{1}}^{1}(\mathscr{D})}+\|\cdot\|_{L_{p_{2}}(\mathscr{D})}$. The dual of $V$ can be identified with the linear space

$$
V^{*}=\left\{f=f_{1}+f_{2}: f_{1} \in W_{p_{1}}^{1}(\mathscr{D})^{*}, f_{2} \in L_{p_{2}}(\mathscr{D})^{*}\right\}
$$

equipped with the norm

$$
\begin{aligned}
\|f\|_{V *} & =\inf \left\{\max \left(\left\|f_{1}\right\|_{W_{p_{1}}^{1}(\mathscr{D})^{*}},\left\|f_{2}\right\|_{L_{p_{2}}(\mathscr{D})^{*}}\right):\right. \\
f & \left.=f_{1}+f_{2}, f_{1} \in W_{p_{1}}^{1}(\mathscr{D})^{*}, \quad f_{2} \in L_{p_{2}}(\mathscr{D})^{*}\right\} .
\end{aligned}
$$

One would then need to show that $\|u\|_{V}\left\|\nabla\left(|\nabla u|^{p_{1}-2} \nabla u\right)+|u|^{p_{2}-2} u\right\|_{V^{*}}$ is locally integrable. To ensure this in general we need, in particular, that

$$
\|u\|_{W_{p_{1}}^{1}(\mathscr{D})}\left\||u|^{p_{2}-2} u\right\|_{L_{p_{2}}(\mathscr{D})^{*}}=\|u\|_{W_{p_{1}}^{1}(\mathscr{D})}\|u\|_{L_{p_{2}}(\mathscr{D})}^{p_{2}-1}
$$

is locally integrable, which we may not have if $p_{1}<p_{2}$. Thus one cannot apply the Itô formula from Gyöngy and Krylov. On the other hand it is easy to check that the assumptions of Theorem 2.1 are satisfied.

An application of the above Itô's formula to SPDEs driven by Wiener processes is given in [14] (Chapter 2, Example 5.1) and in [8]. Further examples can be found in [13, Chapter 2, Section 1.7].

\section{Preliminaries}

Lemma 3.1 For $r \in[0, \infty)$ let $\beta(r):=\inf \{t \geq 0: A(t) \geq r\}$ and let $x(t)$ be a real valued process that is locally integrable with respect to $d A$ for all $\omega \in \Omega$. Then

(i) $\beta(r)$ is a stopping time (not necessarily finite) for every $r \in[0, \infty)$,

(ii)

$$
\begin{aligned}
& \int_{(0, t]} x(s) d A(s)=\int_{(0, A(t)]} x(\beta(r)) d r, \\
& \int_{(0, t)} x(s) d A(s)=\int_{(0, A(t-))]} x(\beta(r)) d r
\end{aligned}
$$

(iii)

for every $t \in[0, \infty)$,

$$
A(\beta(t)-)-A(\beta(s)) \leq t-s
$$


for every $s, t \in[0, \infty)$.

(iv) If $0=r_{0}^{n}<r_{1}^{n}<\cdots<r_{k}^{n}<\cdots$ is an increasing sequence of decompositions of $[0, \infty)$ such that $\sup _{k}\left|r_{k+1}^{n}-r_{k}^{n}\right| \rightarrow 0$ as $n \rightarrow \infty$ then for every $t \geq 0$ and $\omega \in \Omega$

$$
\sum_{k}\left|X\left(\tau_{k+1}^{n} \wedge t\right)-X\left(\tau_{k}^{n} \wedge t\right)\right|^{2} \rightarrow \sum_{s \leq t}|X(s)|^{2}|\Delta A(s)|^{2}
$$

as $n \rightarrow \infty$, where $X(t):=\int_{(0, t]} x(s) d A(s)$ and $\tau_{k}^{n}:=\beta\left(r_{k}^{n}\right)$.

This Lemma is proved in Gyöngy and Krylov [6, Lemma 1].

Let $\kappa_{n}^{(j)}$ for $j=1,2$ and integers $n \geq 1$ denote the functions defined by

$$
\kappa_{n}^{(1)}(t)=2^{-n}\left\lfloor 2^{n} t\right\rfloor, \quad \kappa_{n}^{(2)}(t)=2^{-n}\left\lceil 2^{n} t\right\rceil
$$

The following lemma is known and the authors believe is due to Doob.

Lemma 3.2 For integers $i \geq 1$ let $\left(X_{i},\|\cdot\|_{X_{i}}\right)$ be Banach spaces, and let $p_{i} \in[1, \infty)$. Let $x_{i}: \mathbb{R} \times \Omega \rightarrow X_{i}$ be $\mathscr{B}(\mathbb{R}) \otimes \mathcal{F}$ Bochner-measurable such that $x_{i}(r)=0$ for $r \notin[0,1]$ and

$$
\alpha_{i}:=\mathbb{E} \int_{0}^{1}\left\|x_{i}(r)\right\|_{X_{i}}^{p_{i}} d r<\infty .
$$

Then there exists a subsequence $n_{k} \rightarrow \infty$ such that for dt-almost all $t \in[0,1]$

$$
\mathbb{E} \int_{(0,1]}\left\|x_{i}(r)-x_{i}\left(\kappa_{n_{k}}^{(j)}(r-t)+t\right)\right\|_{X_{i}}^{p_{i}} d r \rightarrow 0 \text { as } k \rightarrow \infty
$$

for $j=1,2$ and all $i \geq 1$.

Proof Let $\left(c_{i}\right)_{i=1}^{\infty}$ be a sequence of positive numbers such that

$$
\sum_{i=1}^{\infty} c_{i} 2^{p_{i}} \alpha_{i}<\infty .
$$

By change of variables and changing the order of integration

$$
\begin{aligned}
I_{n} & :=\sum_{i=1}^{\infty} c_{i} \int_{0}^{1} \mathbb{E} \int_{0}^{1}\left\|x_{i}(r)-x_{i}\left(\kappa_{n}^{(j)}(r-t)+t\right)\right\|_{X_{i}}^{p_{i}} d r d t \\
& \leq \sum_{i=1}^{\infty} c_{i} \mathbb{E} \int_{-1}^{1} \int_{0}^{1}\left\|x_{i}(s+t)-x_{i}\left(\kappa_{n}^{(j)}(s)+t\right)\right\|_{X_{i}}^{p_{i}} d t d s .
\end{aligned}
$$

Note that by the shift invariance of the Lebesgue measure

$$
J_{i n}(s):=\int_{0}^{1}\left\|x_{i}(s+t)-x_{i}\left(\kappa_{n}^{(j)}(s)+t\right)\right\|_{X_{i}}^{p_{i}} d t \rightarrow 0(\text { a.s. })
$$


for $s \in(0,1), i \geq 1$, and

$$
\begin{aligned}
\sum_{i=1}^{\infty} c_{i}\left|J_{i n}(s)\right| & \leq \sum_{i=1}^{\infty} c_{i} 2^{p_{i}-1}\left(\int_{0}^{1}\left\|x_{i}(s+t)\right\|_{X_{i}}^{p_{i}} d t+\int_{0}^{1}\left\|x_{i}\left(\kappa_{n}(s)+t\right)\right\|_{X_{i}}^{p_{i}} d t\right) \\
& \leq \sum_{i=1}^{\infty} c_{i} 2^{p_{i}} \int_{0}^{1}\left\|x_{i}(t)\right\|_{X_{i}}^{p_{i}} d t
\end{aligned}
$$

Therefore by Lebesgue's theorem on dominated convergence

$$
I_{n}=\int_{0}^{1}\left(\sum_{i=1}^{\infty} c_{i} \mathbb{E} \int_{0}^{1}\left\|x_{i}(r)-x_{i}\left(\kappa_{n}^{(j)}(r-t)+t\right)\right\|_{X_{i}}^{p_{i}} d r\right) d t \rightarrow 0
$$

Hence for a subsequence $n_{k} \rightarrow \infty$

$$
\sum_{i=1}^{\infty} c_{i} \mathbb{E} \int_{0}^{1}\left\|x_{i}(r)-x_{i}\left(\kappa_{n}^{(j)}(r-t)+t\right)\right\|_{X_{i}}^{p_{i}} d r \rightarrow 0
$$

for almost all $t \in[0,1]$, and the statement of the lemma follows.

The following lemma is proved in Gyöngy and Krylov [6, Lemma 3].

Lemma 3.3 Let $\left(\xi_{n}\right)_{n \in \mathbb{N}}$ be a sequence of $H$-valued predictable processes. Suppose

$$
\mathbb{P}\left[\sup _{n \in \mathbb{N}, t \leq T}\left|\xi_{n}(t)\right|<\infty\right]=1
$$

and

$$
\mathbb{P}\left[\forall t \leq T, \forall \varphi \in H \quad \lim _{n \rightarrow \infty}\left(\xi_{n}(t), \varphi\right)=0\right]=1 .
$$

Then for any $\varepsilon>0$

$$
\mathbb{P}\left[\sup _{t \leq T}\left|\int_{(0, t]}\left(\xi_{n}(s), d h(s)\right)\right|>\varepsilon\right] \rightarrow 0
$$

as $n \rightarrow \infty$.

\section{Proof of the main result}

The following standard steps, as in Krylov and Rozovskii [12], allow us to work under more convenient assumptions without any loss of generality.

1. We note that $\tau$ can be assumed to be a bounded stopping time. Indeed if we prove Theorem 2.1 under this assumption then we can extend it to unbounded stopping times by considering $\tau \wedge n$ and letting $n \rightarrow \infty$. In fact using a non-random time change we may assume that $\tau \leq 1$. 
2. Recall the processes $\eta_{i}$ from assumption (2.1), and set

$$
Q_{i}(t)=\left(\int_{(0, t]} \eta_{i}^{q_{i}}(s) d A(s)\right)^{1 / q_{i}} t \geq 0
$$

when $q_{i}<\infty$, and for $q_{i}=\infty$ let $Q_{i}=\left(Q_{i}(t)\right)_{t \geq 0}$ denote a nondecreasing cadlag adapted process such that almost surely

$$
d A \text {-ess } \sup _{s \leq t} \eta_{i}(s) \leq Q_{i}(t) \text { for all } t \geq 0 \text {. }
$$

It is not difficult to see that such a process $Q_{i}$ exists, we can take, e.g., the adapted right-continuous modification of the process $d A-\operatorname{ess} \sup _{s \leq t} \eta_{i}(s)$, i.e.,

$$
\lim _{n \rightarrow \infty} d A \text {-ess } \sup _{s \leq t+1 / n} \eta_{i}(s)
$$

Let $\left(e^{j}\right)_{j \in \mathbb{N}} \subset V$ be an orthonormal basis in $H$ and define

$$
\begin{aligned}
& r(t):=|h(0)|+A(t)+\sum_{i=1}^{m}\left(\int_{(0, t]}\|v(s)\|_{V_{i}}^{p_{i}} d A(s)\right)^{1 / p_{i}} \\
& +\sum_{i=1}^{m} Q_{i}(t)+\sum_{i=1}^{m} \sum_{k \in \mathbb{N}} 2^{-c_{k}}\left(\int_{(0, t]}\left\|w_{k}(s)\right\|_{V_{i}}^{p_{i}} d A(s)\right)^{1 / p_{i}},
\end{aligned}
$$

with $c_{k}:=\max _{1 \leq i \leq m} \sum_{j \leq k}\left|e_{j}\right|_{V_{i}}^{2}$ and $w_{k}:=\Pi^{k} h$, where $\Pi^{k}$ denotes the orthogonal projection of $H$ onto its subspace spanned by $\left(e_{i}\right)_{i=1}^{k}$. We may and will assume, without loss of generality, that $r$ and $\langle h\rangle$ are bounded. Indeed, imagine we have proved Theorem 2.1 under this assumption. Consider

$$
\tau_{n}:=\inf \{t \geq 0: r(t) \geq n\}
$$

Then $\tau_{n}$ is a stopping time and $\tau_{n} \rightarrow \infty$ for $n \rightarrow \infty$. Since $\langle h\rangle$ is a predictable process starting from 0 , there is an increasing sequence of stopping times $\sigma_{n}$ such that $\sigma_{n} \rightarrow \infty$ and $\langle h\rangle_{t} \leq n$ for $t \in\left[0, \sigma_{n}\right]$. Therefore $\tau_{n} \wedge \sigma_{n} \wedge \tau \rightarrow \tau$ as $n \rightarrow \infty$, and for fixed $n$ we get $r(t) \leq n$ for $t \in\left(0, \tau_{n} \wedge \sigma_{n}\right)$ and $\langle h\rangle_{t} \leq n$ for $t \in\left[0, \tau_{n} \wedge \sigma_{n}\right]$. Thus we get (2.3) and (2.4) for the stopping time $\tau_{n} \wedge \sigma_{n} \wedge \tau$ in place of $\tau$. Letting $n \rightarrow \infty$ provides (2.3) and (2.4) for $\tau$. Thus we may assume that there is $n \geq 1$ such that $r(t) \leq n$ for $t \in(0, \tau)$ and $\langle h\rangle_{t} \leq n$ for $t \in[0, \tau]$. Moreover, by taking $h \mathbf{1}_{|h(0)|<n}, v \mathbf{1}_{|h(0)|<n}$ and $A \mathbf{1}_{|h(0)|<n}$ in place of $h, v$ and $A$, respectively, and then taking $n \rightarrow \infty$, we may assume that $r(t) \leq n$ for $t \in[0, \tau)$ and $\langle h\rangle_{t} \leq n$ for $t \in[0, \tau]$. Furthermore, we can define $A(t):=A(\tau-), h(t)=h(\tau), v(t)=0$ and $v_{i}^{*}(t)=0$ for $t \geq \tau$. Then $r(t) \leq n$ and $\langle h\rangle_{t} \leq n$ for $t \in[0, \infty)$.

3. Finally, we can assume that $r(t) \leq 1$ for $t \in[0, \tau)$ and $\langle h\rangle_{t} \leq 1$ for $t \in[0, \tau]$. Indeed let $v_{n}:=n^{-1} v, A_{n}:=n^{-1} A$ and $h_{n}:=n^{-1} h$. Then $r_{n}$, defined analogously to $r$ in (4.1) but with $v, A$ and $h$ replaced by $v_{n}, A_{n}$ and $h_{n}$ respectively, 
satisfies $r_{n}(t) \leq n^{-1} r(t) \leq 1$. We thus get (2.3) and (2.4) with $v, A$ and $h$ replaced by $v_{n}, A_{n}$ and $h_{n}$ respectively. We can now multiply by $n$ and $n^{2}$ to obtain the desired conclusions.

Now we proceed to prove Theorem 2.1 under the assumption that $\tau \leq 1, r(t) \leq 1$ and $\langle h\rangle_{t} \leq 1$ for $t \in[0, \infty)$. Our approach is the same as in Gyöngy and Krylov [6]. The idea is to approximate $v$ by simple processes whose jumps happen at stopping times where Eq. (2.2) holds. But (2.2) only holds for every $\varphi \in V$ and $d A \times \mathbb{P}$ almost all $(t, \omega) \in \rrbracket 0, \tau \llbracket$, and thus it is not immediately clear how to choose an appropriate piecewise constant approximation to $v$. Here and later on for stopping times $\tau$ the notation $\rrbracket 0, \tau \llbracket$ means the stochastic interval $\{(t, \omega): t \in(0, \tau(\omega)), \omega \in \Omega\}$.

Proposition 4.1 There is a nested sequence of random partitions of $[0, \infty]$,

$$
0=\tau_{0}^{n}<\tau_{1}^{n} \leq \tau_{2}^{n} \leq \cdots \leq \tau_{N(n)+1}^{n}=\infty
$$

with stopping times $\tau_{j}^{n}, j=1, \ldots, N(n)+1$, such that for every $\omega \in \Omega$ either $\tau_{j}^{n}(\omega)<\tau(\omega)$ or $\tau_{j}^{n}(\omega)=\infty$, and such that the following statements hold.

(1) There is $\Omega^{\prime} \subset \Omega$ such that $\mathbb{P}\left(\Omega^{\prime}\right)=1$ and with

$$
I(\omega):=\left\{\tau_{j}^{n}(\omega): n \in \mathbb{N}, j=1, \ldots, N(n)\right\} \cap(0, \infty)
$$

we have (2.2) satisfied for every $\omega \in \Omega^{\prime}, t \in I(\omega)$ and $\varphi \in V$. Moreover, if $\Delta A(t)>0$ for some $t>0$ and $\omega \in \Omega^{\prime}$, then $t \in I(\omega)$. Furthermore, if $0 \leq s<t$ and $(s, t] \cap I(\omega)=\emptyset$, then $A(s)=A(t)$.

(2) For $l \in\{1,2\}, i=1, \ldots, m$ and for all $k \geq 1$

$$
\begin{array}{r}
\lim _{n \rightarrow \infty} \mathbb{E} \int_{(0, \infty)}\left\|v(s)-v_{n}^{(l)}(s)\right\|_{V_{i}}^{p_{i}} d A(s)=0, \\
\lim _{n \rightarrow \infty} \mathbb{E} \int_{(0, \infty)}\left\|w_{k}(s)-w_{k n}^{(l)}(s)\right\|_{V_{i}}^{p_{i}} d A(s)=0,
\end{array}
$$

where

$$
v_{n}^{(1)}(t):=\sum_{j=1}^{N(n)} v\left(\tau_{j}^{n}\right) \mathbf{1}_{\left[\tau_{j}^{n}, \tau_{j+1}^{n}\right)}(t), \quad v_{n}^{(2)}(t):=\sum_{j=0}^{N(n)} v\left(\tau_{j+1}^{n}\right) \mathbf{1}_{\left(\tau_{j}^{n}, \tau_{j+1}^{n}\right]}(t),
$$

and $w_{k n}^{(l)}$ is defined analogously from $w_{k}=\Pi^{k} h$

Proof Since $V$ is separable there is $\left\{\varphi_{i}\right\}_{i \in \mathbb{N}} \subset V$ which is dense in $V$. For each $\varphi_{i}$ there is an exceptional set $D_{i} \in[0, \infty) \times \Omega$ such that (2.2) holds for $(t, \omega) \in \rrbracket 0, \tau \llbracket \backslash D_{i}$ and $(d A \times \mathbb{P})\left(D_{i}\right)=0$. Let $D=\bigcup_{i \in \mathbb{N}} D_{i}$. Then $(d A \times \mathbb{P})(D)=0$ and (2.2) holds for 
all $\varphi \in V$ and all $(t, \omega) \in \rrbracket 0, \tau \llbracket \backslash D$. Now using Lemma 3.1 and the Fubini theorem

$$
\begin{aligned}
0 & =\mathbb{E} \int_{(0, \tau)} \chi_{D}(s) d A(s)=\mathbb{E} \int_{(0, A(\tau-)]} \chi_{D}(\beta(r)) d r \\
& =\int_{(0, \infty)} \mathbb{P}(r \leq A(\tau),(\beta(r), \omega) \in D) d r .
\end{aligned}
$$

From this we see that for $d r$ almost all $r \in(0, \infty)$ there is $\Omega(r) \subset \Omega$ with $\mathbb{P}(\Omega(r))=$ 1 such that for any $\omega \in \Omega(r)$ either $r>A(\tau(\omega), \omega)$ or $\beta(r, \omega)<\tau(\omega)$ and for $t=\beta(r)$ and for all $\varphi \in V$

$$
(v(t), \varphi)=\sum_{i=1}^{m} \int_{(0, t]}\left\langle v_{i}^{*}(s), \varphi\right\rangle d A(s)+(h(t), \varphi)
$$

By virtue of Lemma 3.2 there is a nested sequence of decompositions of $[0,1]$,

$$
0=r_{0}^{n}<r_{1}^{n}<\cdots<r_{N(n)+1}^{n}=1
$$

such that $\lim _{n \rightarrow \infty} \max _{i}\left|r_{j+1}^{n}-r_{j}^{n}\right|=0$, and

$$
\begin{array}{r}
\lim _{n \rightarrow \infty} \mathbb{E} \int_{0}^{1}\left\|v(\beta(r))-v\left(\beta\left(\kappa_{n}^{(l)}(r)\right)\right)\right\|_{V_{i}}^{p_{i}} d r=0, \\
\lim _{n \rightarrow \infty} \mathbb{E} \int_{0}^{1}\left\|w_{k}(\beta(r))-w_{k}\left(\beta\left(\kappa_{n}^{(l)}(r)\right)\right)\right\|_{V_{i}}^{p_{i}} d r=0
\end{array}
$$

for all $i=1, \ldots, m$, all $k \in \mathbb{N}$ and $l=1,2$, where $\kappa_{n}^{(1)}(r)=r_{j}^{n}$ if $r \in\left[r_{j}^{n}, r_{j+1}^{n}\right)$ and $\kappa_{n}^{(2)}(r)=r_{j+1}^{n}$ if $r \in\left(r_{j}^{n}, r_{j+1}^{n}\right]$.

Now let $\Omega^{\prime}:=\bigcap_{n \in \mathbb{N}}\left(\Omega\left(r_{0}^{n}\right) \cap \ldots \cap \Omega\left(r_{N(n)+1}^{n}\right)\right), \tau_{j}^{n}:=\beta\left(r_{j}^{n}\right)$, and

$$
I(\omega):=\left\{\tau_{i}^{n}(\omega): n \in \mathbb{N}, i=1, \ldots, N(n)\right\} \cap(0, \infty) .
$$

Then $\mathbb{P}\left(\Omega^{\prime}\right)=1$ and

$$
0=\tau_{0}^{n}<\tau_{1}^{n} \leq \tau_{2}^{n} \leq \cdots \leq \tau_{N(n)+1}^{n}=\infty, \quad n=1,2, \ldots,
$$

is a nested sequence of random partitions of $(0,1)$ by stopping times $\tau_{j}^{n}$ such that statement (1) holds. To prove (2) we notice that, just like in [6], for $r \in\left(r_{j}^{n}, r_{j+1}^{n}\right.$ ]

$$
v_{n}^{(2)}(\beta(r))=\left\{\begin{array}{l}
v\left(\beta\left(r_{j+1}^{n}\right)\right)=v\left(\beta\left(\kappa_{n}^{(2)}(r)\right)\right) \text { if } \beta\left(r_{j}^{n}\right)<\beta(r) \\
v\left(\beta\left(r_{j}^{n}\right)\right)=v\left(\beta\left(\kappa_{n}^{(1)}(r)\right)\right) \text { if } \beta\left(r_{j}^{n}\right)=\beta(r) .
\end{array}\right.
$$


Thus with appropriate sets $S_{n} \in \mathcal{B}(\mathbb{R}) \times \mathcal{F}$

$$
\left.v^{(2)}(\beta(r))=\mathbf{1}_{S_{n}}(r) v\left(\beta\left(\kappa_{n}^{(2)}(r)\right)\right)-\left(1-\mathbf{1}_{S_{n}}\right)(r)\right) v\left(\beta\left(\kappa_{n}^{(1)}(r)\right)\right) .
$$

Hence due to (4.5) and Lemma 3.1 we obtain the first equality in (4.2) for $l=2$, $i=1, \ldots, m$ and for all $k \in \mathbb{N}$. The rest of (4.2) is obtained similarly.

Proposition 4.2 For every $n \in \mathbb{N}$, every $\omega \in \Omega^{\prime}$ and every $\tau_{j}^{n}(\omega) \in I(\omega)$

$$
\begin{aligned}
\left|v\left(\tau_{j}^{n}\right)\right|^{2}= & |h(0)|^{2}+2 \sum_{i=1}^{m} \int_{\left(0, \tau_{j}^{n}\right]}\left\langle v_{i}^{*}(s), v_{n}^{(2)}(s)\right\rangle d A(s) \\
& +2 \int_{\left(0, \tau_{j}^{n}\right]} \bar{v}_{n}(s) d h(s)+2\left(h(0), h\left(\tau_{1}^{n}\right)-h(0)\right) \\
& +\sum_{k=0}^{j-1}\left|h\left(\tau_{k+1}^{n}\right)-h\left(\tau_{k}^{n}\right)\right|^{2}-\left|v\left(\tau_{1}^{n}\right)-h\left(\tau_{1}^{n}\right)\right|^{2} \\
& -\sum_{k=1}^{j-1}\left|v\left(\tau_{k+1}^{n}\right)-v\left(\tau_{k}^{n}\right)-\left(h\left(\tau_{k+1}^{n}\right)-h\left(\tau_{k}^{n}\right)\right)\right|^{2},
\end{aligned}
$$

where $\bar{v}_{n}(s)=0$ for $s \in\left[0, \tau_{1}^{n}\right]$ and $\bar{v}_{n}(s)=v\left(\tau_{j}^{n}\right)$ for $s \in\left(\tau_{j}^{n}, \tau_{j+1}^{n}\right]$ for $j=$ $1, \ldots, N(n)$. Moreover,

$$
\mathbb{E} \sup _{t \in I}|v(t)|^{2}<\infty
$$

Proof Let $\omega \in \Omega^{\prime}$ and $t, t^{\prime} \in I(\omega)$ and $t^{\prime} \geq t$. Clearly,

$$
\left|v\left(t^{\prime}\right)\right|^{2}-|v(t)|^{2}=2\left(v\left(t^{\prime}\right), v\left(t^{\prime}\right)-v(t)\right)-\left|v\left(t^{\prime}\right)-v(t)\right|^{2}
$$

which by statement (1) of Proposition 4.1 gives

$$
\begin{aligned}
& \left|v\left(t^{\prime}\right)\right|^{2}-|v(t)|^{2} \\
& \quad=2 \sum_{i=1}^{m} \int_{\left(t, t^{\prime}\right]}\left\langle v_{i}^{*}(s), v\left(t^{\prime}\right)\right\rangle d A(s)+2\left(h\left(t^{\prime}\right)-h(t), v\left(t^{\prime}\right)\right)-\left|v\left(t^{\prime}\right)-v(t)\right|^{2} .
\end{aligned}
$$

Hence by the identity

$$
\begin{aligned}
& 2\left(h\left(t^{\prime}\right)-h(t), v\left(t^{\prime}\right)-v(t)\right) \\
& \quad=-\left|v\left(t^{\prime}\right)-v(t)-\left(h\left(t^{\prime}\right)-h(t)\right)\right|^{2}+\left|v\left(t^{\prime}\right)-v(t)\right|^{2}+\left|h\left(t^{\prime}\right)-h(t)\right|^{2},
\end{aligned}
$$


we have

$$
\begin{gathered}
\left|v\left(t^{\prime}\right)\right|^{2}-|v(t)|^{2}=2 \sum_{i=1}^{m} \int_{\left(t, t^{\prime}\right]}\left\langle v_{i}^{*}(s), v\left(t^{\prime}\right)\right\rangle d A(s)+2\left(v(t), h\left(t^{\prime}\right)-h(t)\right) \\
+\left|h\left(t^{\prime}\right)-h(t)\right|^{2}-\left|v\left(t^{\prime}\right)-v(t)-\left(h\left(t^{\prime}\right)-h(t)\right)\right|^{2} .
\end{gathered}
$$

By (1) in Proposition 4.1 again

$$
2|v(t)|^{2}=2 \sum_{i=1}^{m} \int_{(0, t]}\left\langle v_{i}^{*}(s), v(t)\right\rangle d A(s)+2(h(t), v(t)),
$$

which by the identity $2(h(t), v(t))=-|v(t)-h(t)|^{2}+|v(t)|^{2}+|h(t)|^{2}$ gives

$$
|v(t)|^{2}=2 \sum_{i=1}^{m} \int_{(0, t]}\left\langle v_{i}^{*}(s), v(t)\right\rangle d A(s)+|h(t)|^{2}-|v(t)-h(t)|^{2} .
$$

Summing up for $k=1, \ldots, j-1$ equations (4.8) with $t^{\prime}=\tau_{k+1}^{n}, t=\tau_{k}^{n}$, and adding to it Eq. (4.9) with $t=\tau_{1}^{n}$, we obtain (4.6). Form (4.6) we have

$$
\begin{aligned}
\mathbb{E} \max _{1 \leq j \leq N(n)}\left|v\left(\tau_{j}^{n}\right)\right|^{2} \leq & 2 \mathbb{E}|h(0)|^{2}+2 \mathbb{E} \sum_{i=1}^{m} \int_{(0, \tau]}\left|\left\langle v_{i}^{*}(s), v_{n}^{(2)}(s)\right\rangle\right| d A(s) \\
& +2 \mathbb{E} \max _{1 \leq j \leq N(n)}\left|\int_{\left(0, \tau_{j}^{n}\right]} \bar{v}_{n}(s) d h(s)\right| \\
& +2 \mathbb{E} \sum_{k=0}^{N(n)}\left|h\left(\tau_{k+1}^{n}\right)-h\left(\tau_{k}^{n}\right)\right|^{2}
\end{aligned}
$$

Clearly

$$
2 \mathbb{E} \max _{1 \leq j \leq N(n)}\left|\int_{\left(0, \tau_{j}^{n}\right]} \bar{v}_{n}(s) d h(s)\right| \leq 16+\frac{1}{16} \mathbb{E} \sup _{t \geq 0}\left|\int_{(0, t]} \bar{v}_{n}(s) d h(s)\right|^{2},
$$

and by Doob's inequality and $\langle h\rangle \leq 1$,

$$
\mathbb{E} \sup _{t \geq 0}\left|\int_{(0, t]} \bar{v}_{n}(s) d h(s)\right|^{2} \leq 4 \mathbb{E} \int_{0}^{\infty}\left|\bar{v}_{n}(s)\right|^{2} d\langle h\rangle_{s} \leq 4 \mathbb{E} \max _{1 \leq j \leq N(n)}\left|v\left(\tau_{j}^{n}\right)\right|^{2} .
$$

Since $h$ is a martingale,

$$
\mathbb{E} \sum_{k=0}^{N(n)}\left|h\left(\tau_{k+1}^{n}\right)-h\left(\tau_{k}^{n}\right)\right|^{2} \leq \mathbb{E}|h(1)|^{2}=\mathbb{E}\langle h\rangle(1) \leq 1
$$


By Hölder's inequality and $\sum_{i} Q_{i} \leq 1$ we have

$$
\begin{aligned}
& \sum_{i=1}^{m} \mathbb{E} \int_{(0, \tau]}\left|\left\langle v_{i}^{*}(s), v_{n}^{(2)}(s)\right\rangle\right| d A(s) \\
& \quad \leq \sum_{i} \sup _{n \geq 1}\left(\mathbb{E} \int_{(0, \tau]}\left\|v_{n}^{(2)}(s)\right\|_{V_{i}}^{p_{i}} d A(s)\right)^{\frac{1}{p_{i}}}=: c,
\end{aligned}
$$

which by virtue of (4.2) is finite. Hence, taking also into account $\mathbb{E}|h(0)|^{2} \leq 1$ we have

$$
\mathbb{E} \max _{1 \leq j \leq N(n)}\left|v\left(\tau_{j}^{n}\right)\right|^{2} \leq 22+2 c+\frac{1}{4} \mathbb{E} \max _{1 \leq j \leq N(n)}\left|v\left(\tau_{j}^{n}\right)\right|^{2},
$$

which immediately yields (4.7), provided

$$
\mathbb{E} \max _{1 \leq j \leq N(n)}\left|v\left(\tau_{j}^{n}\right)\right|^{2}<\infty
$$

To show (4.10) note that due to (4.9), for every $n \in \mathbb{N}$ and $j=1, \ldots, N(n)+1$, we get

$$
\begin{aligned}
\mathbb{E}\left|v\left(\tau_{j}^{n}\right)\right|^{2} & \leq \mathbb{E}\left|h\left(\tau_{j}^{n}\right)\right|^{2}+2 \mathbb{E} \sum_{i=1}^{m} \int_{\left(0, \tau_{j}^{n}\right]}\left\langle v_{i}^{*}(s), v\left(\tau_{j}^{n}\right)\right\rangle d A(s) \\
& \leq \mathbb{E}|h(0)|^{2}+2 \mathbb{E} \sum_{i=1}^{m} Q_{i}(\tau)\left(\int_{(0, \tau]}\left\|v\left(\tau_{j}^{n}\right)\right\|_{V_{i}}^{p_{i}} d A(s)\right)^{\frac{1}{p_{i}}} \\
& \leq 1+2 \sum_{i} \mathbb{E}\left\|v\left(\tau_{j}^{n}\right)\right\|_{V_{i}},
\end{aligned}
$$

since $\tau \leq 1$ and $r(t) \leq 1$ for all $t \in[0, \infty)$. For $i=1, \ldots, m$

$$
\begin{aligned}
& \mathbb{E}\left\|v\left(\tau_{j}^{n}\right)\right\|_{V_{i}}^{p_{i}} \leq \mathbb{E} \sup _{s \in[0, \infty)}\left\|v_{n}^{(2)}(s)\right\|_{V_{i}}^{p_{i}} \leq \mathbb{E} \sup _{r \in(0,1]}\left\|v_{n}^{(2)}(\beta(r))\right\|_{V_{i}}^{p_{i}} \\
& \leq 2^{p_{i}-1} \sum_{l=1}^{2} \mathbb{E} \sup _{r \in(0,1]}\left\|v\left(\beta\left(\kappa_{n}^{(l)}(r)\right)\right)\right\|_{V_{i}}^{p_{i}} \\
& \leq 2^{p_{i}-1} \sum_{l=1}^{2} \mathbb{E} \sum_{k=0}^{N(n)} \frac{1}{r_{k+1}^{n}-r_{k}^{n}} \int_{r_{k}^{n}}^{r_{k+1}^{n}}\left\|v\left(\beta\left(\kappa_{n}^{(l)}(r)\right)\right)\right\|_{V_{i}}^{p_{i}} d r \\
& \leq \frac{2^{p_{i}-1}}{d_{n}} \sum_{l=1}^{2} \mathbb{E} \int_{0}^{1}\left\|v\left(\beta\left(\kappa_{n}^{(l)}(r)\right)\right)\right\|_{V_{i}}^{p_{i}} d r<2^{p_{i}} \frac{c_{i}}{d_{n}},
\end{aligned}
$$


where $r_{k}^{n}$ are given by (4.4), $d_{n}:=\min _{k=1, \ldots, N(n)}\left|r_{k+1}^{n}-r_{k}^{n}\right|>0$ and

$$
c_{i}:=\max _{l} \sup _{n} \sum_{i=1}^{m} \mathbb{E} \int_{0}^{1}\left\|v\left(\beta\left(\kappa_{n}^{(l)}(r)\right)\right)\right\|_{V_{i}}^{p_{i}} d r
$$

which due to (4.5) is finite. Hence by virtue of (4.11) we have (4.10), which completes the proof of (4.7).

We see that due to (4.7) there is $\Omega^{\prime \prime} \subset \Omega^{\prime}$ such that $\mathbb{P}\left(\Omega^{\prime \prime}\right)=1$ and

$$
\sup _{t \in I(\omega)}|v(t)|^{2}<\infty \text { for all } \omega \in \Omega^{\prime \prime} .
$$

Moreover, since $h$ is cadlag, for all $\omega \in \Omega^{\prime \prime}$ we have

$$
\sup _{t \in I(\omega)}|v(t)-h(t)|^{2}<\infty
$$

Define

$$
z^{(1)}(t):=\int_{(0, t)} \sum_{i=1}^{m} v_{i}^{*}(s) d A(s), \quad z^{(2)}(t):=\int_{(0, t]} \sum_{i=1}^{m} v_{i}^{*}(s) d A(s),
$$

for $t \geq 0$, where the integrals are defined as weak* integrals. Recall that $v^{*}=\sum_{i} v^{*}$ is a $V^{*}$-valued such that $\left\langle v^{*}(t), \varphi\right\rangle$ is a progressively measurable process for every $\varphi \in V$, and

$$
\begin{aligned}
& \int_{(0, t]}\left|\left\langle v^{*}(s), \varphi\right\rangle\right| d A(s) \leq \sum_{i} \int_{(0, t]}\left|\left\langle v_{i}^{*}(s), \varphi\right\rangle\right| d A(s) \\
& \quad \leq \sum_{i}|\varphi|_{V_{i}} \int_{(0, t]} \eta_{i}(s) d A(s) \leq|\varphi|_{V} \sum_{i} \int_{(0, t]} \eta_{i}(s) d A(s)<\infty .
\end{aligned}
$$

Therefore $z^{(1)}$ and $z^{(2)}$ are well-defined $V^{*}$-valued processes such that $\left\langle z^{(1)}, \varphi\right\rangle$ and $\left\langle z^{(2)}, \varphi\right\rangle$ are left-continuous and right-continuous adapted processes, respectively.

In what follows we use the notation $\Delta^{w} f(t):=f(t)-\mathrm{w}-\lim _{s}{ }_{t} f(s)$ for $H$-valued functions $f$, when the weak limit from the left exists at $t$.

Proposition 4.3 Let $z^{(l)}, l \in\{1,2\}$ be given by (4.13).

1. If $\omega \in \Omega^{\prime \prime}$ and $t \in(0, \infty)$ then $z^{(l)}(t) \in H$ for $l \in\{1,2\}$. Moreover

$$
\sup _{t \in(0, \infty)}\left|z^{(l)}(t)\right|<\infty \quad \forall \omega \in \Omega^{\prime \prime}, l \in\{1,2\}
$$

2. Let $\tilde{v}$ be given by

$$
\tilde{v}(t):=\chi \Omega_{\Omega^{\prime \prime}} z^{(2)}(t)+h(t) .
$$

Then $\tilde{v}$ is a $H$-valued adapted and weakly cadlag process such that $v(t)=\tilde{v}(t)$ for all $t \in I(\omega)$ and $\omega \in \Omega^{\prime \prime}$. Moreover 


$$
\sup _{t \in(0, \infty)}|\tilde{v}(t)|<\infty \quad \forall \omega \in \Omega^{\prime \prime}
$$

3. If $\omega \in \Omega^{\prime \prime}$ then for all $t \in(0, \tau(\omega))$

$$
\Delta^{w}(\tilde{v}-h)(t)=(\Delta A)(t) \sum_{i=1}^{m} v_{i}^{*}(t)
$$

Proof Fix $\omega \in \Omega^{\prime \prime}$. If $t \in I(\omega)$ then for all $\varphi \in V$

$$
(v(t)-h(t), \varphi)=\sum_{i=1}^{m} \int_{(0, t]}\left\langle v_{i}^{*}(s), \varphi\right\rangle d A(s),
$$

and hence $z^{(2)}(t) \in H$. Consider now the situation when $t \in(0, \tau(\omega)] \backslash I(\omega)$. Let $\bar{I}^{l}(\omega)$ denote the left-closure of the set $I(\omega)$. If $t \in \bar{I}^{l}(\omega) \backslash I(\omega)$ then $\Delta A(t)=0$ by Proposition 4.1, and there is a sequence $\left(t_{n}\right)_{n \in \mathbb{N}} \subset I(\omega)$ such that $t_{n} \nearrow t$. Moreover, due to (4.12) there is a subsequence $t_{n^{\prime}} \nearrow t$ such that $v\left(t_{n^{\prime}}\right)-h\left(t_{n^{\prime}}\right)$ converges weakly in $H$ to some $\xi \in H$. Hence for all $\varphi \in V$

$$
\begin{aligned}
(\xi, \varphi) & =\lim _{n^{\prime} \rightarrow \infty}\left(v\left(t_{n^{\prime}}\right)-h\left(t_{n^{\prime}}\right), \varphi\right) \\
& =\lim _{n^{\prime} \rightarrow \infty} \sum_{i=1}^{m} \int_{\left(0, t_{n^{\prime}}\right]}\left\langle v_{i}^{*}(s), \varphi\right\rangle d A(s)=\sum_{i=1}^{m} \int_{(0, t)}\left\langle v_{i}^{*}(s), \varphi\right\rangle d A(s) \\
& =\sum_{i=1}^{m} \int_{(0, t]}\left\langle v_{i}^{*}(s), \varphi\right\rangle d A(s)
\end{aligned}
$$

which implies $z^{(2)}(t)=\xi \in H$. If $t \in(0, \infty) \backslash \bar{I}^{l}(\omega)$, then there is $s \in\{0\} \cup \bar{I}^{l}(\omega)$ such that $s<t$ and $(s, t] \cap I(\omega)=\emptyset$. So $\int_{(s, t]} v_{i}^{*}(s) d A(s)=0$ and $z^{(2)}(t)=z^{(2)}(s) \in H$. Of course if $t=0$ then $z^{(2)}(t)=0 \in H$. Finally, due to (4.12),

$$
\sup _{t \in(0, \infty)}\left|z^{(2)}(t)\right|^{2}=\sup _{t \in(0, \infty)}|v(t)-h(t)|^{2}<\infty
$$

Now we consider $z^{(1)}(t)$ for $t \in(0, \infty)$. Take $\left(t_{n}\right)_{n \in \mathbb{N}}$ such that $t_{n}<t$ and $t_{n} \nearrow t$ as $n \rightarrow \infty$. From (4.15) we know that $\sup _{n \in \mathbb{N}}\left|z^{(2)}\left(t_{n}\right)\right|^{2}<\infty$ and so there is a subsequence $t_{n^{\prime}} \nearrow t$ such that $z^{(2)}\left(t_{n}\right)$ converges weakly in $H$ to some $\xi \in H$. Thus for any $\varphi \in V$

$$
\begin{aligned}
& (\xi, \varphi)=\lim _{n^{\prime} \rightarrow \infty}\left(z^{(2)}\left(t_{n^{\prime}}\right), \varphi\right) \\
& =\lim _{n^{\prime} \rightarrow \infty} \sum_{i=1}^{m} \int_{\left(0, t_{n^{\prime}}\right]}\left\langle v_{i}^{*}(s), \varphi\right\rangle d A(s)=\sum_{i=1}^{m} \int_{\left(0, t_{n^{\prime}}\right)}\left\langle v_{i}^{*}(s), \varphi\right\rangle d A(s)=\left\langle z^{(1)}(t), \varphi\right\rangle .
\end{aligned}
$$


Hence $z^{(1)}(t)=\xi \in H$, and due to (4.15)

$$
\sup _{t \in(0, \infty)}\left|z^{(1)}(t)\right|^{2} \leq \sup _{t \in(0, \infty)}\left|z^{(2)}(t)\right|^{2}<\infty .
$$

By construction $\tilde{v}$ is weakly cadlag. Due to (4.15) for $\omega \in \Omega^{\prime \prime}$

$$
\sup _{t \in(0, \infty)}|\tilde{v}(t)|^{2} \leq \sup _{t \in(0, \infty)}\left|z^{(2)}(t)\right|^{2}+\sup _{t \in(0, \infty)}|h(t)|^{2}<\infty .
$$

We note that for any $\varphi \in V$ the real valued random variable

$$
(\tilde{v}(t), \varphi)=\chi_{\Omega^{\prime \prime}} \sum_{i=1}^{m} \int_{(0, t]}\left\langle v_{i}^{*}(s), \varphi\right\rangle d A(s)+(h(t), \varphi)
$$

is $\mathcal{F}_{t}$-measurable. Hence, since $H$ is separable, $\tilde{v}(t)$ is $\mathcal{F}_{t}$-measurable by the Pettis theorem. Finally notice that

$$
\Delta((\tilde{v}-h)(t), \varphi)=\sum_{i=1}^{m}\left\langle v_{i}^{*}(t), \varphi\right\rangle(\Delta A)(t)
$$

for all $\varphi \in V$ and $\omega \in \Omega^{\prime \prime}$. Hence on $\Omega^{\prime \prime}$

$$
\Delta^{w}(\tilde{v}-h)(t)=\sum_{i=1}^{m} v_{i}^{*}(t)(\Delta A)(t)
$$

Let

$$
\tilde{v}_{n}(t):=\tilde{v}\left(\tau_{j}^{n}\right) \text { and } h_{n}(t):=h\left(\tau_{j}^{n}\right) \text { for } t \in\left(\tau_{j}^{n}, \tau_{j+1}^{n}\right], \quad j=0,1, \ldots, N(n)
$$

Then from (4.6) it follows that for every $\omega \in \Omega^{\prime \prime}$ and $t:=\tau_{j}^{n}(\omega) \in I(\omega)$

$$
\begin{aligned}
|\tilde{v}(t)|^{2}= & |h(0)|^{2}+2 \sum_{i=1}^{m} \int_{(0, t]}\left\langle v_{i}^{*}(s), v_{n}^{(2)}(s)\right\rangle d A(s) \\
& +2 \int_{(0, t]}\left(\tilde{v}_{n}(s), d h(s)\right)+\sum_{k=0}^{j-1}\left|h\left(\tau_{k+1}^{n}\right)-h\left(\tau_{k}^{n}\right)\right|^{2}-K_{n}(t),
\end{aligned}
$$

where

$$
K_{n}(t):=\sum_{k: \tau_{k+1}^{n} \leq t}^{j-1}\left|\tilde{v}\left(\tau_{k+1}^{n}\right)-\tilde{v}\left(\tau_{k}^{n}\right)-\left(h\left(\tau_{k+1}^{n}\right)-h\left(\tau_{k}^{n}\right)\right)\right|^{2}
$$


In order to let $n \rightarrow \infty$ in the above equation we first rewrite it as

$$
\begin{aligned}
|\tilde{v}(t)|^{2}= & 2 \sum_{i=1}^{m} \int_{(0, t]}\left\langle v_{i}^{*}(s), v_{n}^{(2)}(s)\right\rangle d A(s) \\
& +2 \int_{(0, t]}\left(\tilde{v}_{n}(s)-h_{n}(s), d h(s)\right)+|h(t)|^{2}-K_{n}(t)
\end{aligned}
$$

by noticing that

$$
2 \int_{\left(0, \tau_{j}^{n}\right]}\left(h_{n}(s), d h(s)\right)=\left|h\left(\tau_{j}^{n}\right)\right|^{2}-|h(0)|^{2}-\sum_{k=0}^{j-1}\left|h\left(\tau_{k+1}^{n}\right)-h\left(\tau_{j}^{n}\right)\right|^{2} .
$$

To perform the limit procedure we use the following two propositions.

Proposition 4.4 There is $\tilde{\Omega} \subset \Omega^{\prime \prime}$ with $\mathbb{P}(\tilde{\Omega})=1$ such that for a subsequence $n^{\prime}$ and for every $\omega \in \tilde{\Omega}$

$$
\begin{aligned}
& \int_{(0, \infty)}\left\|v(s)-v_{n^{\prime}}^{(l)}(s)\right\|_{V_{i}}^{p_{i}} d A(s) \rightarrow 0 \quad(l=1,2), \\
& \int_{(0, \infty)}\left\|w_{k}(s)-w_{k n^{\prime}}^{(l)}(s)\right\|_{V_{i}}^{p_{i}} d A(s) \rightarrow 0 \quad(l=1,2 ; k \in \mathbb{N}), \\
& \sup _{t \in(0, \infty)}\left|\int_{(0, t]}\left(\tilde{v}_{n^{\prime}}(s)-h_{n^{\prime}}(s), d h(s)\right)-\int_{(0, t]}(\tilde{v}(s-)-h(s-), d h(s))\right| \rightarrow 0
\end{aligned}
$$

as $n^{\prime} \rightarrow \infty$. Moreover,

$$
K_{n^{\prime}}(t) \rightarrow \int_{(0, t]}\left|v^{*}(s)\right|^{2} \Delta A(s) d A(s) \text { for } t \in I(\omega) \text { and } \omega \in \tilde{\Omega}
$$

Proof Set $\xi(t):=\tilde{v}(t-)-h(t-)$ and $\xi_{n}(t):=\tilde{v}_{n}(t)-h_{n}(t)$. By Lemma 3.3, taking into account that by Proposition 4.3 on $\Omega^{\prime \prime}$

$$
\sup _{n} \sup _{t \in(0, \infty)}\left|\xi(t)-\xi_{n}(t)\right| \leq \sup _{t \in(0, \infty)}\left|z^{(1)}(t)\right|<\infty
$$

and that $V$ is dense in $H$, we have

$$
\sup _{t \geq 0}\left|\int_{(0, t]}\left(\xi(s)-\xi_{n}(s), d h(s)\right)\right| \rightarrow 0 \text { in probability as } n \rightarrow \infty
$$

if we show that almost surely

$$
\lim _{n \rightarrow \infty}\left(\xi(t)-\xi_{n}(t), \varphi\right)=0 \text { for all } t>0 \text { and } \varphi \in V
$$


To this end set

$$
v_{i}^{*}:=\int_{\left(\tau_{j}^{n}, t\right)} v_{i}^{*}(s) d A(s) \in V_{i}^{*} .
$$

Then for all $\omega \in \Omega^{\prime \prime}, t>0$ and $\varphi \in V$

$$
\begin{aligned}
& \left(\xi(t)-\xi_{n}(t), \varphi\right)=\left\langle\xi(t)-\xi_{n}(t), \varphi\right\rangle=\left\langle\sum_{i=1}^{m} v_{i}^{*}, \varphi\right\rangle=\sum_{i=1}^{m}\left\langle v_{i}^{*}, \varphi\right\rangle \\
& \quad=\sum_{i=1}^{m} \int_{\left(\tau_{j}^{n}, t\right)}\left\langle v_{i}^{*}(s), \varphi\right\rangle d A(s) \leq \sum_{i=1}^{m}\|\varphi\|_{V_{i}} \int_{\left(\tau_{j}^{n}, t\right)}\left\|v_{i}^{*}(s)\right\|_{V_{i}^{*}} d A(s) \\
& \quad \leq \max _{j=1, \ldots, N(n)} \sum_{i=1}^{m}\|\varphi\|_{V_{i}}\left(A\left(\tau_{j+1}^{n}\right)-A\left(\tau_{j}^{n}\right)\right)^{\frac{1}{p_{i}}} Q_{i}\left(\tau_{j+1}^{n}\right) \\
& \quad \leq \max _{j=1, \ldots, N(n)} \sum_{i=1}^{m}\|\varphi\|_{V_{i}}\left|r_{j+1}^{n}-r_{j}^{n}\right|^{\frac{1}{p_{i}}} \rightarrow 0 \text { as } n \rightarrow \infty,
\end{aligned}
$$

with $r_{j}^{n}$ given by (4.4). Consequently, taking also into account (4.2) of Proposition 4.1 we have $\Omega^{\prime \prime \prime} \subset \Omega^{\prime \prime}$ and a subsequence $n^{\prime}$ such that the first three limits are zero for $\omega \in \Omega^{\prime \prime \prime}$. Taking the limit along the subsequence $n^{\prime}$ in (4.17) we see that $K_{n^{\prime}}(t)$ converges for $\omega \in \Omega^{\prime \prime \prime}$ and $t \in I(\omega)$ to some $K(t)$, and

$$
\begin{aligned}
|\tilde{v}(t)|^{2}= & 2 \sum_{i=1}^{m} \int_{(0, t]}\left\langle v_{i}^{*}(s), v(s)\right\rangle d A(s) \\
& +2 \int_{(0, t]}(\tilde{v}(s)-h(s), d h(s))+|h(t)|^{2}-K(t) .
\end{aligned}
$$

From this point onwards we will always consider only the subsequence $n^{\prime}$ but we will keep writing $n$ to ease notation. Our task is now to identify $K(t)$. We note that, using Parseval's identity,

$$
\begin{aligned}
K_{n}(t) & =\sum_{0 \leq \tau_{j+1}^{n} \leq t}\left|\sum_{i} \int_{\left(\tau_{j}^{n}, \tau_{j+1}^{n}\right]} v_{i}^{*}(s) d A(s)\right|^{2} \\
& =\sum_{0 \leq \tau_{j+1}^{n} \leq t} \sum_{k \in \mathbb{N}}\left(\sum_{i} \int_{\left(\tau_{j}^{n}, \tau_{j+1}^{n}\right]} v_{i}^{*}(s) d A(s), e_{k}\right)^{2} \\
& =\sum_{0 \leq \tau_{j+1}^{n} \leq t} \sum_{k \in \mathbb{N}}\left\langle\sum_{i} \int_{\left(\tau_{j}^{n}, \tau_{j+1}^{n}\right]} v_{i}^{*}(s) d A(s),\left.e_{k}\right|^{2}\right. \\
& =\sum_{0 \leq \tau_{j+1}^{n} \leq t} \sum_{k \in \mathbb{N}}\left|\int_{\left(\tau_{j}^{n}, \tau_{j+1}^{n}\right]} \sum_{i}\left\langle v_{i}^{*}(s), e_{k}\right\rangle d A(s)\right|^{2} .
\end{aligned}
$$


Hence, using Lemma 3.1, Parseval's identity and (4.14), we get

$$
\begin{aligned}
K(t) & =\lim _{n \rightarrow \infty} K_{n}(t) \geq \sum_{k \in \mathbb{N}} \underset{n \rightarrow \infty}{\lim _{n \rightarrow \tau_{j+1}^{n} \leq t}}\left|\int_{\left(\tau_{j}^{n}, \tau_{j+1}^{n}\right]} \sum_{i}\left\langle v_{i}^{*}(s), e_{k}\right\rangle d A(s)\right|^{2} \\
& =\sum_{k \in \mathbb{N}} \sum_{s \leq t}\left|\sum_{i}\left\langle v_{i}^{*}(s), e_{k}\right\rangle \Delta A(s)\right|^{2}=\sum_{k \in \mathbb{N}} \sum_{s \leq t}\left|\left(\Delta^{w}(\tilde{v}-h)(s), e_{k}\right)\right|^{2} \\
& =\sum_{s \leq t}\left|\sum_{i} v_{i}^{*}(s)\right|^{2}|\Delta A(s)|^{2} .
\end{aligned}
$$

To obtain an upper bound we use first the identity

$$
|x+y|^{2}=y^{2}+2 x(y+x)-x^{2}
$$

together with the definition of $g$ to get

$$
\begin{aligned}
K_{n}(t) & =\sum_{0 \leq \tau_{j+1}^{n} \leq t}\left|\sum_{i} \int_{\left(\tau_{j}^{n}, \tau_{j+1}^{n}\right)} v_{i}^{*}(s) d A(s)+\sum_{i} v_{i}^{*}\left(\tau_{j+1}^{n}\right) \Delta A\left(\tau_{j+1}^{n}\right)\right|^{2} \\
& =\sum_{0 \leq \tau_{j+1}^{n} \leq t}\left(J_{j}^{(1)}+J_{j}^{(2)}-J_{j}^{(3)}\right)
\end{aligned}
$$

with

$$
\begin{aligned}
& J_{j}^{(1)}:=\left|\sum_{i} v_{i}^{*}\left(\tau_{j+1}^{n}\right)\right|^{2}\left|\Delta A\left(\tau_{j+1}^{n}\right)\right|^{2}, \\
& J_{j}^{(2)}:=2\left(\sum_{i} \int_{\left(\tau_{j}^{n}, \tau_{j+1}^{n}\right)} v_{i}^{*}(s) d A(s), \tilde{v}\left(\tau_{j+1}^{n}\right)-\tilde{v}\left(\tau_{j}^{n}\right)-\left(h\left(\tau_{j+1}^{n}\right)-h\left(\tau_{j}^{n}\right)\right)\right), \\
& J_{j}^{(3)}:=\left|\sum_{i} \int_{\left(\tau_{j}^{n}, \tau_{j+1}^{n}\right)} v_{i}^{*}(s) d A(s)\right|^{2}
\end{aligned}
$$

For $j \neq 0$ we split $J_{j}^{(2)}=J_{j}^{(21)}-J_{j}^{(22)}$ with

$$
\begin{aligned}
J_{j}^{(21)} & :=2\left(\sum_{i} \int_{\left(\tau_{j}^{n}, \tau_{j+1}^{n}\right)} v_{i}^{*}(s) d A(s), \tilde{v}\left(\tau_{j+1}^{n}\right)-\tilde{v}\left(\tau_{j}^{n}\right)\right), \\
J_{j}^{(22)} & :=2\left(\sum_{i} \int_{\left(\tau_{j}^{n}, \tau_{j+1}^{n}\right)} v_{i}^{*}(s) d A(s), h\left(\tau_{j+1}^{n}\right)-h\left(\tau_{j}^{n}\right)\right),
\end{aligned}
$$


and notice that

$$
\begin{aligned}
J_{j}^{(21)} & =2 \sum_{i} \int_{\left(\tau_{j}^{n}, \tau_{j+1}^{n}\right)}\left\langle v_{i}^{*}(s), v\left(\tau_{j+1}^{n}\right)-v\left(\tau_{j}^{n}\right)\right\rangle d A(s) \\
& =2 \sum_{i} \int_{\left[\tau_{j}^{n}, \tau_{j+1}^{n}\right)}\left\langle v_{i}^{*}(s), v_{n}^{(2)}(s)-v_{n}^{(1)}(s)\right\rangle d A(s) .
\end{aligned}
$$

Using $\Pi_{k}$, the orthogonal projection of $H$ onto the space spanned by $\left(e_{j}\right)_{j=1}^{k} \subset V$, we have

$$
J_{j}^{(22)}=J_{j k}^{(22)}+\bar{J}_{j k}^{(22)}
$$

with

$$
\begin{aligned}
J_{j k}^{(22)} & :=2\left(\sum_{i} \int_{\left(\tau_{j}^{n}, \tau_{j+1}^{n}\right)} v_{i}^{*}(s) d A(s), \Pi_{k}\left(h\left(\tau_{j+1}^{n}\right)-h\left(\tau_{j}^{n}\right)\right)\right), \\
\bar{J}_{j k}^{(22)} & :=2\left(\sum_{i} \int_{\left(\tau_{j}^{n}, \tau_{j+1}^{n}\right)} v_{i}^{*}(s) d A(s),\left(I-\Pi_{k}\right)\left(h\left(\tau_{j+1}^{n}\right)-h\left(\tau_{j}^{n}\right)\right)\right) .
\end{aligned}
$$

Notice that

$$
\begin{aligned}
J_{j k}^{(22)} & =2 \sum_{i} \int_{\left(\tau_{j}^{n}, \tau_{j+1}^{n}\right)}\left\langle v_{i}^{*}(s), \Pi_{k}\left(h\left(\tau_{j+1}^{n}\right)-h\left(\tau_{j}^{n}\right)\right)\right\rangle d A(s), \\
& =2 \sum_{i} \int_{\left[\tau_{j}^{n}, \tau_{j+1}^{n}\right)}\left\langle v_{i}^{*}(s), w_{k n}^{(2)}(s)-w_{k n}^{(1)}(s)\right\rangle d A(s),
\end{aligned}
$$

and

$$
\bar{J}_{j k}^{(22)} \leq J_{j}^{(3)}+\left|\left(I-\Pi_{k}\right)\left(h\left(\tau_{j+1}^{n}\right)-h\left(\tau_{j}^{n}\right)\right)\right|^{2} .
$$

Similarly, taking into account $\tilde{v}(0)=h(0)$, for $J_{0}^{(2)}$ we have

$$
J_{0}^{(2)}=2\left(\sum_{i} \int_{\left(0, \tau_{1}^{n}\right)} v_{i}^{*}(s) d A(s), \tilde{v}\left(\tau_{1}^{n}\right)-h\left(\tau_{1}^{n}\right)\right)=J_{0}^{(21)}-J_{0}^{(22)} \text {, }
$$

where

$$
\begin{aligned}
J_{0}^{(21)} & :=2 \sum_{i} \int_{\left(0, \tau_{1}^{n}\right)}\left\langle v_{i}^{*}(s), \tilde{v}\left(\tau_{1}^{n}\right)\right\rangle d A(s) \\
& =2 \sum_{i} \int_{\left(0, \tau_{1}^{n}\right)}\left\langle v_{i}^{*}(s), v^{(2)}(s)-v^{(1)}(s)\right\rangle d A(s),
\end{aligned}
$$


and

$$
J_{0}^{(22)}:=2\left(\sum_{i} \int_{\left(0, \tau_{1}^{n}\right)} v_{i}^{*}(s) d A(s), h\left(\tau_{1}^{n}\right)\right)=J_{0 k}^{(22)}+\bar{J}_{0 k}^{(22)}
$$

with

$$
\begin{aligned}
J_{0 k}^{(22)} & :=\sum_{i} \int_{\left(0, \tau_{1}^{n}\right)}\left\langle v_{i}^{*}(s), w^{(2)}(s)-w^{(1)}(s)\right\rangle d A(s), \\
\bar{J}_{0 k}^{(22)} & :=2\left(\sum_{i} \int_{\left(0, \tau_{1}^{n}\right)} v_{i}^{*}(s) d A(s),\left(I-\Pi_{k}\right) h\left(\tau_{1}^{n}\right)\right) \\
& \leq J_{0}^{(3)}+\left|\left(I-\Pi_{k}\right) h\left(\tau_{1}^{n}\right)\right|^{2} .
\end{aligned}
$$

Thus from (4.19) we get

$$
\begin{aligned}
K_{n}(t) \leq & \sum_{0 \leq \tau_{j+1}^{n} \leq t}\left|\sum_{i} v_{i}^{*}\left(\tau_{j+1}^{n}\right)\right|^{2}\left|\Delta A\left(\tau_{j+1}^{n}\right)\right|^{2} \\
& +2 \sum_{i} \int_{(0, t)}\left\langle v_{i}^{*}(s), v_{n}^{(2)}(s)-v_{n}^{(1)}(s)\right\rangle d A(s) \\
& -2 \sum_{i} \int_{(0, t)}\left\langle v_{i}^{*}(s), w_{n k}^{(2)}(s)-w_{n k}^{(1)}(s)\right\rangle d A(s)+\xi_{n k}(t)
\end{aligned}
$$

with

$$
\xi_{n k}(t):=\sum_{j=1}^{N(n)}\left|\left(I-\Pi_{k}\right)\left(h\left(\tau_{j+1}^{n} \wedge t\right)-h\left(\tau_{j}^{n} \wedge t\right)\right)\right|^{2}+\left|\left(I-\Pi_{k}\right) h\left(\tau_{1}^{n} \wedge t\right)\right|^{2}
$$

for every $n, k \in \mathbb{N}$. As $n \rightarrow \infty$ we see that

$$
\sum_{0 \leq \tau_{j+1}^{n} \leq t}\left|\sum_{i} v_{i}^{*}\left(\tau_{j+1}^{n}\right)\right|^{2}\left|\Delta A\left(\tau_{j+1}^{n}\right)\right|^{2} \rightarrow \sum_{0<s \leq t}\left|v^{*}(s)\right|^{2}|\Delta A(s)|^{2},
$$

where we use the notation $v^{*}(s)=\sum_{i} v_{i}^{*}(s)$. By Hölder's inequality, taking into account $r(t) \leq 1$, we have

$$
\begin{aligned}
& \varlimsup_{n \rightarrow \infty} \int_{(0, t)}\left|\left\langle v_{i}^{*}(s), v_{n}^{(2)}(s)-v_{n}^{(1)}(s)\right\rangle\right| d A(s) \\
& \leq \varlimsup_{n \rightarrow \infty}\left(\int_{(0, t)}\left\|v_{n}^{(2)}(s)-v_{n}^{(1)}(s)\right\|_{V_{i}}^{p_{i}} d A(s)\right)^{\frac{1}{p_{i}}}=0
\end{aligned}
$$


and similarly,

$$
\varlimsup_{n \rightarrow \infty} \int_{(0, t)}\left|\left\langle v_{i}^{*}(s), w_{n k}^{(2)}(s)-w_{n k}^{(1)}(s)\right\rangle\right| d A(s)=0
$$

for all integers $k \geq 1$ and $i=1,2, \ldots, m$. Thus

$$
K(t)=\varliminf_{n \rightarrow \infty} K_{n}(t) \leq \sum_{s \leq t}\left|v^{*}(s)\right|^{2}|\Delta A(s)|^{2}+\xi_{k}(t)
$$

for every $k \in N$, where

$$
\begin{aligned}
\xi_{k}(t):=\lim _{n \rightarrow \infty}\left(\sum_{j=1}^{N(n)}\left|\left(I-\Pi_{k}\right)\left(h\left(\tau_{j+1}^{n} \wedge t\right)-h\left(\tau_{j}^{n} \wedge t\right)\right)\right|^{2}\right. \\
\left.+\left|\left(I-\Pi_{k}\right) h\left(\tau_{1}^{n} \wedge t\right)\right|^{2}\right) .
\end{aligned}
$$

Note that by Fatou's lemma and the martingale property of $h$

$$
\mathbb{E} \xi_{k}(t) \leq \mathbb{E}\left|\left(I-\Pi_{k}\right) h(1)\right|^{2} \rightarrow 0 \text { as } k \rightarrow \infty .
$$

Note also that for each $\omega \in \Omega$ and $t \in(0, \infty)$ we have $\xi_{k} \geq \xi_{k+1}$ and $\xi_{k} \geq 0$. Thus there exists a set $\Omega^{\prime \prime \prime \prime} \subset \Omega$ with $P\left(\Omega^{\prime \prime \prime \prime}\right)=1$ such that for every $t \in[0, \infty)$ and $\omega \in \Omega^{\prime \prime \prime \prime}$ we have $\xi_{k}(t) \rightarrow 0$. Letting here $k \rightarrow \infty$ in (4.20) we obtain

$$
K(t) \leq \int_{(0, t]}\left|v^{*}(s)\right|^{2} \Delta A(s) d A(s),
$$

which together with (4.18) gives

$$
K(t)=\int_{(0, t]}\left|v^{*}(s)\right|^{2} \Delta A(s) d A(s)
$$

for $\omega \in \tilde{\Omega}:=\Omega^{\prime \prime \prime} \cap \Omega^{\prime \prime \prime \prime}$ and $t \in I(\omega)$.

Proposition 4.5 For $\omega \in \tilde{\Omega}$

$$
\begin{aligned}
& |\tilde{v}(t)|^{2}=|h(0)|^{2}+2 \sum_{i=1}^{m} \int_{(0, t]}\left\langle v_{i}^{*}(s), v(s)\right\rangle d A(s) \\
& \quad+2 \int_{(0, t]}(\tilde{v}(s-), d h(s))-\int_{(0, t]}\left|\sum_{i=1}^{m} v_{i}^{*}(s)\right|^{2} \Delta A(s) d A(s)+[h]_{t}
\end{aligned}
$$

for $t \in[0, \tau(\omega))$. 
Proof Let $\omega \in \tilde{\Omega}$ be fixed and let $t \in[0, \tau(\omega))$. To ease notation we use $n \rightarrow \infty$ in place of the subsequence $n^{\prime} \rightarrow \infty$ defined in the previous proposition. If $t \in I(\omega)$, then by virtue of the previous proposition taking $n \rightarrow \infty$ in (4.17) we obtain

$$
\begin{aligned}
|\tilde{v}(t)|^{2}= & |h(t)|^{2}+2 \sum_{i=1}^{m} \int_{(0, t]}\left\langle v_{i}^{*}(s), v(s)\right\rangle d A(s)+2 \int_{(0, t]}(\tilde{v}(s-)-h(-), d h(s)) \\
& -\int_{(0, t]}\left|\sum_{i=1}^{m} v_{i}^{*}(s)\right|^{2} \Delta A(s) d A(s) .
\end{aligned}
$$

Hence using the Itô formula for Hilbert space valued processes

$$
|h(t)|^{2}=|h(0)|^{2}+2 \int_{(0, t]}(h(s-), d h(s))+[h]_{t},
$$

we get (4.21) for $t \in I(\omega)$. If $t \in \bar{I}^{l}(\omega) \backslash I(\omega)$, then for sufficiently large $n$ there is $j=j(n)$ such that $t_{n}:=\tau_{j}^{n}(\omega) \in I(\omega)$ and $t_{n} \nearrow t$ for $n \rightarrow \infty$. Using the algebraic relationship

$$
|\tilde{v}(s)-\tilde{v}(r)|^{2}=|\tilde{v}(s)|^{2}-|\tilde{v}(r)|^{2}-2(\tilde{v}(r), \tilde{v}(s)-\tilde{v}(r)),
$$

with $s:=t_{n}, r:=t_{l}$, and since (4.21) holds for every $t \in I(\omega)$, we get

$$
\begin{aligned}
& \left|\tilde{v}\left(t_{n}\right)-\tilde{v}\left(t_{l}\right)\right|^{2}=2 \sum_{i=1}^{m} \int_{\left(t_{l}, t_{n}\right]}\left\langle v_{i}^{*}(s), v(s)\right\rangle d A(s)+2 \int_{\left(t_{l}, t_{n}\right]}(\tilde{v}(s-), d h(s)) \\
& \quad-\int_{\left(t_{l}, t_{n}\right]}\left|\sum_{i=1}^{m} v_{i}^{*}(s)\right|^{2} \Delta A(s) d A(s)+[h]_{t_{n}}-[h]_{t_{l}}-2\left(\tilde{v}\left(t_{l}\right), \tilde{v}\left(t_{n}\right)-\tilde{v}\left(t_{l}\right)\right)
\end{aligned}
$$

for $n>l$. Moreover

$$
\begin{aligned}
& 2\left(\tilde{v}\left(t_{l}\right), \tilde{v}\left(t_{n}\right)-\tilde{v}\left(t_{l}\right)\right) \\
& \quad=2 \sum_{i=1}^{m} \int_{\left(t_{l}, t_{n}\right]}\left\langle v_{i}^{*}(s), v\left(t_{l}\right)\right\rangle d A(s)+2\left(\tilde{v}\left(t_{l}\right), h\left(t_{n}\right)-h\left(t_{l}\right)\right) .
\end{aligned}
$$


Hence by (4.22)

$$
\begin{aligned}
\left|\tilde{v}\left(t_{n}\right)-\tilde{v}\left(t_{l}\right)\right|^{2}= & 2 \sum_{i=1}^{m} \int_{\left(t_{l}, t_{n}\right]}\left\langle v_{i}^{*}(s), v(s)-v\left(t_{l}\right)\right\rangle d A(s) \\
& +2 \int_{\left(t_{l}, t_{n}\right]}\left(\tilde{v}(s-)-h(s-)-\left(\tilde{v}\left(t_{l}\right)-h\left(t_{l}\right)\right), d h(s)\right) \\
& -\int_{\left(t_{l}, t_{n}\right]}\left|\sum_{i=1}^{m} v_{i}^{*}(s)\right|^{2} \Delta A(s) d A(s)+\left|h\left(t_{n}\right)-h\left(t_{l}\right)\right|^{2} \\
= & : 2 I_{l n}^{1}+2 I_{l n}^{2}-I_{l n}^{3}+I_{l n}^{4} .
\end{aligned}
$$

Since $h$ is cadlag we have

$$
\lim _{l \rightarrow \infty} \sup _{n>l} I_{l n}^{4}=\lim _{l \rightarrow \infty} \sup _{n>l}\left|h\left(t_{n}\right)-h\left(t_{l}\right)\right|^{2}=0 .
$$

By the previous proposition we get

$$
\begin{aligned}
& \lim _{l \rightarrow \infty} \sup _{n>l}\left|I_{l n}^{2}\right|=\lim _{l \rightarrow \infty} \sup _{n>l}\left|\int_{\left(t_{l}, t_{n}\right]}\left(\tilde{v}(s-)-h(s-)-\left(\tilde{v}_{l}(s)-h_{l}(s)\right), d h(s)\right)\right| \\
& \leq 2 \lim _{l \rightarrow \infty} \sup _{t \in(0, \infty)}\left|\int_{(0, t]}\left(\tilde{v}(s-)-h(s-)-\left(\tilde{v}_{l}(s)-h_{l}(s)\right), d h(s)\right)\right|=0,
\end{aligned}
$$

and

$$
\lim _{l \rightarrow \infty} \sup _{n>l}\left|I_{l n}^{1}\right| \leq \lim _{l \rightarrow \infty} \sum_{i=1}^{m} \int_{(0, \infty)}\left\|v_{i}^{*}(s)\right\|_{V_{i}^{*}}\left\|v(s)-v_{l}^{(1)}(s)\right\|_{V_{i}} d A(s)=0,
$$

via $r(t) \leq 1$ and Hölder's inequality. Thus

$$
\lim _{l \rightarrow \infty} \sup _{n>l}\left|\tilde{v}\left(t_{n}\right)-\tilde{v}\left(t_{l}\right)\right|^{2}=0
$$

and so the sequence $\left(\tilde{v}\left(t_{n}\right)\right)_{n \in \mathbb{N}}$ converges strongly to some $\xi$ in $H$. Moreover since $\tilde{v}$ is weakly cadlag and $t_{n} \nearrow t$, we conclude that $\xi=\tilde{v}(t-)$. Hence using (4.21) with $t_{n}$ in place of $t$, and letting $n \rightarrow \infty$ we obtain

$$
\begin{aligned}
& |\tilde{v}(t-)|^{2}=|h(0)|^{2}+2 \sum_{i=1}^{m} \int_{(0, t)}\left\langle v_{i}^{*}(s), v(s)\right\rangle d A(s) \\
& \quad+2 \int_{(0, t)}(\tilde{v}(s-), d h(s))-\int_{(0, t)}\left|\sum_{i=1}^{m} v_{i}^{*}(s)\right|^{2} \Delta A(s) d A(s)+[h]_{t-}
\end{aligned}
$$

for $t \in I(\omega) \backslash \bar{I}^{l}(\omega)$, and so for this $t$ we get also (4.21) by taking into account that $\Delta A(t)=0$. If $t \in(0, \tau(\omega)) \backslash \bar{I}^{l}(\omega)$, then there is $t^{\prime} \in\{0\} \cup \bar{I}^{l}(\omega)$ such that $t^{\prime}<t$ and 
$\left(t^{\prime}, t\right] \cap I(\omega)=\emptyset$. Thus $d A(s)=0$ for $s \in\left(t^{\prime}, t\right]$, and so $\tilde{v}(s)-\tilde{v}\left(t^{\prime}\right)=h(s)-h\left(t^{\prime}\right)$. Hence applying (4.21) with $t:=t^{\prime}$, and the formula

$$
|\tilde{v}(t)|^{2}-\left|\tilde{v}\left(t^{\prime}\right)\right|^{2}=2\left(\tilde{v}\left(t^{\prime}\right), \tilde{v}(t)-\tilde{v}\left(t^{\prime}\right)\right)+\left|\tilde{v}(t)-\tilde{v}\left(t^{\prime}\right)\right|^{2}
$$

together with the Itô formula for Hilbert space valued martingales,

$$
\left|h(t)-h\left(t^{\prime}\right)\right|^{2}=2 \int_{\left(t^{\prime}, t\right]}\left(h(s-)-h\left(t^{\prime}\right), d h(s)\right)+[h]_{t}-[h]_{t^{\prime}},
$$

we obtain (4.21) for the $t$ under consideration.

Now we can finish the proof of Theorem 2.1 by noting that by the above proposition $|\tilde{v}(t)|^{2}$ is a cadlag process, and since by Proposition 4.3 the process $\tilde{v}$ is $H$-valued and weakly cadlag, it follows by identity (4.23) that $\tilde{v}$ is an $H$-valued cadlag process.

Acknowledgements The authors are sincerely grateful to the anonymous referees. Their corrections and valuable suggestions helped improve the presentation of the paper.

Open Access This article is distributed under the terms of the Creative Commons Attribution 4.0 International License (http://creativecommons.org/licenses/by/4.0/), which permits unrestricted use, distribution, and reproduction in any medium, provided you give appropriate credit to the original author(s) and the source, provide a link to the Creative Commons license, and indicate if changes were made.

\section{References}

1. Da Prato, G., Jentzen, A., Röckner, M.: A mild Itô formula for SPDEs (2016)

2. Dareiotis, K., Gyöngy, I.: A comparison principle for stochastic integro-differential equations. Potential Anal. 41(4), 1203-1222 (2014)

3. Evans, L.C.: Partial Differential Equations. AMS, Providence (1998)

4. Gajewski, H., Gröger, K., Zacharias, K.: Nichtlineare Operatorgleichungen und Operatordifferentialgleichungen. Akademie-Verlag, Berlin (1974)

5. Gyöngy, I., Krylov, N.V.: On stochastic equations with respect to semimartingales I. Stochastics 4(1), 1-21 (1980)

6. Gyöngy, I., Krylov, N.V.: On stochastic equations with respect to semimartingales II. Itô formula in Banach spaces. Stochastics 6(3), 153-173 (1982)

7. Gyöngy, I.: On stochastic equations with respect to semimartingales III. Stochastics 7(4), 231-254 (1982)

8. Gyöngy, I., Sabanis, S., Šiška, D.: Convergence of tamed Euler schemes for a class of stochastic evolution equations. Stoch. Partial Differ. Equ. Anal. Comput. 4(2), 225-245 (2016)

9. Krylov, N.V.: Maximum principle for SPDEs and its applications In: Stochastic Differential Equations: Theory and Applications, Interdisciplinary Mathematical Sciences, vol. 2. World Scientific Publishing Co. Pte. Ltd., Hackensack (2007)

10. Krylov, N.V.: Itô's formula for the $L_{p}$-norm of stochastic $W_{p}^{1}$-valued processes. Probab. Theory Relat. Fields 147, 583-605 (2010)

11. Krylov, N.V.: A relatively short proof of Itô's formula for SPDEs and its applications. Stoch. PDE Anal. Comput. 1, 152-174 (2013)

12. Krylov, N.V., Rozovskii, B.L.: Stochastic evolution equations. J. Sov. Math. 16, 1233-1277 (1981)

13. Lions, J.L.: Quelques méthodes de résolution des problèmes aux limites non linéaires. Dunod GauthierVillars, Paris (1969)

14. Pardoux, E.: Équations aux dériveés partielles stochastiques non linéaries monotones. Étude des solution forte de type Ito. These Université de Paris Sud, Orsay (1975) 
15. Prévôt, C., Röckner, M.: A Concise Course on Stochastic Partial Differential Equations. Lecture Notes in Mathematics, vol. 1905. Springer, Berlin (2007)

16. Rozovskii, B.L.: Stochastic Evolution Systems. Linear Theory and Applications to Nonlinear Filtering. Kluwer, Dordrecht (1990) 